(C) 2012 IEEE. Personal use of this material is permitted. Permission from IEEE must be obtained for all other uses, in any current or future media, including reprinting/republishing this material for advertising or promotional purposes, creating new collective works, for resale or redistribution to servers or lists, or reuse of any copyrighted component of this work in other works. 


\section{A hypoglycemic episode diagnosis system based on neural networks for Type 1 diabetes mellitus}

\author{
Kit Yan Chan \\ Department of Electrical and Computer Engineering, \\ Curtin University, WA, \\ Australia
}

\author{
Sai Ho Ling, H.T. Nguyen \\ Centre for Health Technologies, Faculty of Engineering and \\ Information Technology, University of Technology, Sydney, \\ NSW, Australia \\ Frank Jiang \\ Faculty of Engineering and Information Engineering, \\ University of New South Wales, NSW, \\ Australia
}

\begin{abstract}
Hypoglycemia (or low blood glucose) is dangerous for Type 1 diabetes mellitus (T1DM) patients, as this can cause unconsciousness or even death. However, it is impossible to monitor the hypoglycemia by measuring patients' blood glucose levels all the time, especially at night. In this paper, a hypoglycemic episode diagnosis system is proposed to determine T1DM patients' blood glucose levels based on these patients' physiological parameters which can be measured online. It can be used not only to diagnose hypoglycemic episodes in T1DM patients, but also to generate a set of rules, which describe the domains of physiological parameters that lead to hypoglycemic episodes. The hypoglycemic episode diagnosis system addresses the limitations of the traditional neural network approaches which cannot generate implicit information. The performance of the proposed hypoglycemic episode diagnosis system is evaluated by using real T1DM patients' data sets collected from the Department of Health, Government of Western Australia, Australia. Results show that satisfactory diagnosis accuracy can be obtained. Also, explicit knowledge can be produced such that the deficiency of traditional neural networks can be overcome. A clear understanding of how they perform diagnosis can be indicated.
\end{abstract}

Keywords- hypoglycemic episodes, Type 1 diabetes mellitus, diagnosis system, konwledge discovery system, artifical neural networks, evolutionary algoritms

\section{INTRODUCTION}

Episodes of hypoglycemia for Type 1 diabetes mellitus (T1DM) patients are common in insulin therapy [8]. It can result in unconsciousness, or seizures. More than $50 \%$ of all severe episodes of hypoglycemia occur at night [15], as usual insulin preparations do not adequately mimic the normal patterns of endogenous insulin secretion [19]. It is, however, impossible to monitor the episodes of hypoglycemia by measuring the blood glucose levels around the clock. Therefore, it is necessary to develop a diagnosis model to determine the episodes of hypoglycemia based on some available physiological measures including heart rate and QT interval of ECG signal, which relate to the glucose levels of the T1DM [12].
To develop such diagnosis models, the statistical regression method [18] has been used in the diagnosis of diabetic nephropathy [5], acute gastrointestinal bleeding [6], and pancreatic cancer [4]. However, the modeling of highly nonlinear patients' characteristics, and handling the fuzziness of the physiological measures using statistical regression methods, is still an issue. Neural networks [16] have been used to develop models for medical diagnosis purposes, where both the nonlinear and fuzzy nature of the patients' data can be addressed. Even if they have been applied in building diagnosis models for various diagnoses [1, 3, 7, 10, 13], these models only have the capability to transform the nonlinear or fuzzy patients' data into simplified black-box structures. Hence, no explicit knowledge can be indicated within the neural networks. As the neural networks are black-box in nature, some medical doctors may feel uncomfortable about using them for diagnostic purposes, although the neural networks may achieve better diagnosis accuracy than the other explicit modeling methods such as classical statistical methods. This could pose serious issues if one has to justify a decision based on the implicit outputs of the neural networks. Therefore, it is essential to extract explicit knowledge from the neural networks, so that the basis on which the decision is made is explicit.

In this paper, a hypoglycemic episode diagnosis system, which consists of two units namely diagnosis unit and knowledge discovery unit, is proposed for the diagnosis of hypoglycemic episodes in T1DM patients. First, the diagnosis unit is used to determine hypoglycemic episodes in T1DM patients using the specified physiological parameters. Based on the collected TIDM patients' data, a genetic algorithm, was developed which has a fitness function intended to optimize two goals: a) maximize the number of correctly diagnosed TIDM patients with hypoglycemic episodes during these episodes, and the number of TIDM patients in normal conditions diagnosed correctly in normal conditions; and b) minimize the number of TIDM patients with hypoglycemic episodes diagnosed wrongly under normal conditions, and the number of TIDM patients under normal conditions wrongly 
diagnosed with hypoglycemic episodes. Then, the knowledge discovery unit is used to extract a set of explicit rules, which describe the domains of physiological parameters for which hypoglycemic episodes occur according to the results produced by the diagnosis unit. The explicit rules were generated based on a data set generated by the diagnosis unit. The explicit rules are then validated by a set of testing data, and satisfactory results can also be found. The knowledge discovery system compensates for the limitation of neural networks in not providing explicit information.

The performance of the proposed hypoglycemic episode diagnosis system is evaluated by using real T1DM patients' data sets collected from Department of Health, Government of Western Australia, Australia. Results show that satisfactory diagnosis accuracy can be obtained by the hypoglycemic episode diagnosis system. Also, explicit knowledge can be extracted, thereby addressing the deficiency of traditional neural networks, which do not provide a clear understanding of how diagnosis is performed.

\section{Diagnosis OF HyPoglycemic EPISODES}

Usual insulin preparations do not adequately mimic the normal patterns of endogenous insulin secretion, and so, episodes of hypoglycemia are common at night [19]. Therefore, diagnosis of hypoglycemic episodes is necessary for T1DM patients, especially at night. The blood glucose level of TIDM, $y$, can indicate whether the patients are hypoglycemic. It is significantly related to several physiological parameters, the three most significant of which have been identified as being: i) changes of heart rates, $x_{1}$,; ii) corrected QT interval of electrocardiogram signal, $x_{2}$; ; and iii) rates of changes of corrected QT interval, $x_{3}$.

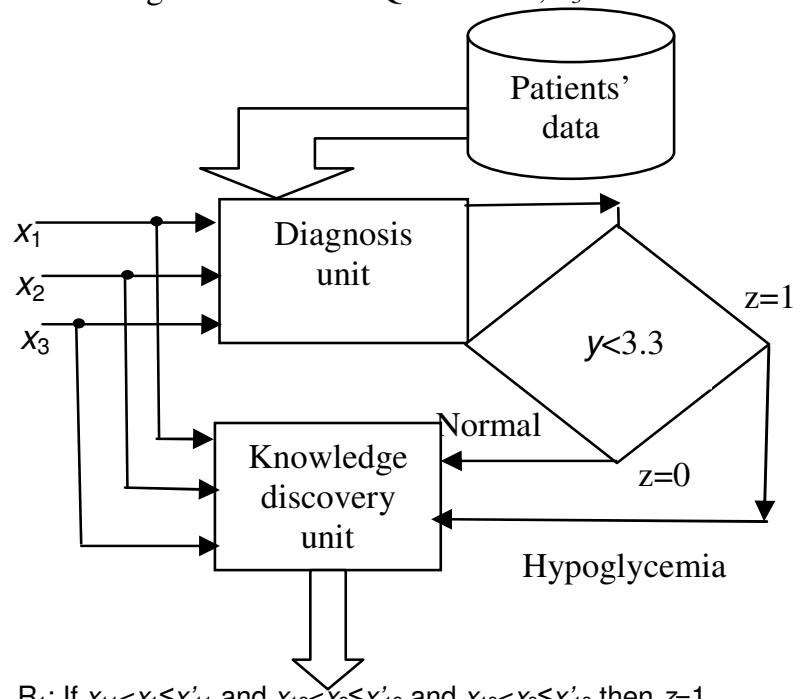

$\mathrm{R}_{1}$ : If $X_{11}<X_{1} \leq x_{11}^{\prime}$ and $x_{12}<x_{2} \leq x_{12}^{\prime}$ and $x_{13}<X_{3} \leq x_{13}^{\prime}$ then $z=1$ OR

$\mathrm{R}_{2}$ : If $x_{21}<x_{1} \leq x_{21}^{\prime}$ and $x_{22}<x_{2} \leq x_{22}^{\prime}$ and $x_{23}<x_{3} \leq x_{23}^{\prime}$ then $z=1$ OR

$\mathrm{R}_{\mathrm{n}}$ : If $x_{\mathrm{n} 1}<x_{1} \leq x_{\mathrm{n} 1}^{\prime}$ and $x_{\mathrm{n} 2}<x_{2} \leq x_{\mathrm{n} 2}^{\prime}$ and $x_{\mathrm{n} 3}<x_{3} \leq x_{\mathrm{n} 3}^{\prime}$ then $z=1$

Figure 1 An illustration of the hypoglycemic episode diagnosis system
Hypoglycemic episodes are those in which the patient's blood glucose level is less than $3.33 \mathrm{mmol} / \mathrm{l}(60 \mathrm{mg} / \mathrm{dl})$ [8, 9]. In this paper, a hypoglycemic episode diagnosis system (shown in Figure 1) is proposed consisting of two main units: a) a diagnosis unit which is used to determine whether the patient is hypoglycemic based on the three most significant physiological parameters, $x_{1}, x_{2}$, and $x_{3}$. It is trained by a genetic algorithm based on a set of patients' data; and b) a knowledge discovery unit which is used to extract knowledge from the diagnosis unit. It is trained by a genetic algorithm based on a set of data generated by the diagnosis unit. This proposed hypoglycemic episode diagnosis system and its two main units are discussed in Section II.A and Section II.B respectively.

\section{A. Diagnosis unit}

The diagnosis unit is developed based on a three-layer feedforward neural network which is used to indicate whether or not the patient is hypoglycemic in relation to the patient's three physiological parameters: rate of change of heart rate, $x_{1}$, corrected QT interval of electrocardiogram signal, $x_{2}$ and rate of change of corrected QT interval, $x_{3}$. It consists of an input layer including the three physiological parameters $x_{1}, x_{2}$ and $x_{3}$ which are fed in, and the output layer which produces the indication of hypoglycemia $z=0$ or 1 . The patient is in normal condition with $z=0$ if the glucose level $y$ of the patient is higher than $3.3 \mathrm{mmol} / \mathrm{l}$. Otherwise, they are in a hypoglycemic state with $z=1$ if the glucose level $y$ of the patient is lower than 3.3 $\mathrm{mmol} / \mathrm{l}$. The hidden layer links together the physiological parameters and the indication of hypoglycemia. They also allow for complex, nonlinear interactions between the three physiological parameters to produce the indication of hypoglycemia. The input-output relationship of the proposed three-layer neural network for the glucose level $y$ is denoted as follows:

$$
y=\sum_{j=1}^{n_{h}} w_{j} \log \operatorname{sig}\left[\sum_{i=1}^{3}\left(v_{i j} x_{i}-b_{j}\right)\right]-b
$$

where $n_{h}$ denotes the number of the hidden nodes; $w_{j}, j=1,2, \ldots$, $n_{h}$, denotes the weight of the link between the $j$-th hidden node and the output; $v_{i j}, i=1,2,3$ and $j=1,2, \ldots, n_{h}$, denotes the weight between the $i$-th input and the $j$-th hidden node; $b_{j}$ and $b$, denote the biases for the $j$-th hidden nodes and output nodes respectively; $\log \operatorname{sig}($.) denotes the logarithmic sigmoid function:

$$
\log \operatorname{sig}(\alpha)=\frac{1}{1+e^{-\alpha}}, \quad \alpha \in \Re
$$

To develop the neural network for the estimation of hypoglycemia, values of the neural network parameters (i.e. $w_{j}$, $v_{i j}, b_{j}$ and $b$ with $i=1,2,3$ and $\left.j=1,2, \ldots, n_{h}\right)$ and the number of hidden-nodes (i.e. $n_{h}$ ) used in the hidden layer need to be determined. These two settings affect not only the convergence of neural networks, but also the accuracy of the estimation of the neural network. Here, a genetic algorithm is used to determine the neural network parameters. The neural network architecture is 3- $n_{h}-1$, denoted as $N N A_{n_{k}}$, where $n_{h}$ is the number of hidden nodes. First the neural network architecture, 
$N N A_{n_{k}}$, is selected and then a neural network is constructed, according to the neural network architecture. After that, the neural network parameters (i.e.: $w_{j}, v_{i j}, b_{j}$ and $b$ with $i=1,2,3$ and $\left.j=1,2, \ldots, n_{h}\right)$ are searched by a genetic algorithm, and finally the trained error of the developed neural network is calculated.

The genetic algorithm first generates a population of strings, randomly represented by the parameters of the neural network with an architecture $N N A_{n_{k}}$. The neural network's parameters, $w_{j}, v_{i j}, b_{j}$ and $b$, are represented by the chromosomes of the genetic algorithms, where $-1 \geq w_{j}, v_{i j}, b_{j}, b \geq 1, i=1,2,3$ and $j=1,2, \ldots, n_{h}$. The length of the strings is equal to the total number of neural network parameters, which is $n_{h}+3 n_{h}+n_{h}+1=5 n_{h}+1$. Then the fitness of each string is evaluated by a fitness function which is defined as:

$$
\text { fitness }=\lambda \varsigma+(1-\lambda) \kappa
$$

where $\varsigma$ and $\kappa$ are the sensitivity and the specificity of the T1DM problem represented by the string respectively, and $\lambda \in$ $\left[\begin{array}{ll}0 & 1\end{array}\right]$ is a constant value to control the importance of the sensitivity and specificity. The objective of the genetic algorithm is to maximize both $\varsigma$ and $\kappa$, which are defined as follows:

$$
\begin{gathered}
\varsigma=\frac{N_{T P}}{N_{T P}+N_{F N}}, \\
\kappa=\frac{N_{T N}}{N_{T N}+N_{F P}},
\end{gathered}
$$

$N_{T P}$ is number of true positives which imply those people correctly diagnosed as being sick; $N_{F N}$ is number of false negatives which imply the sick people wrongly diagnosed as healthy; $N_{F P}$ is the number of false positives which imply healthy people wrongly diagnosed as sick; and $N_{T N}$ is the number of true negatives which imply healthy people correctly diagnosed as healthy [2].

\section{B. Knowledge discovery unit}

It is difficult to extract explicit information from the diagnosis unit based solely on (1) which has an implicit structure, even if the hypoglycemia $z$ of a T1DM can be determined based on the three physiological parameters, $x_{1}, x_{2}$ and $x_{3}$ by using the diagnosis unit discussed in Section II.A. A methodological approach based on a knowledge discovery unit developed by a genetic algorithm is used to extract explicit rules from the diagnosis unit.

Based on the diagnosis unit, the hypoglycemia $z$ of a T1DM can be determined based on the three physiological parameters, $x_{1}, x_{2}$ and $x_{3}$, where $\mathfrak{R}_{1}, \mathfrak{R}_{2}, \ldots$ and $\mathfrak{R}_{n}$ (shown in Figure 2) are the domains regarding the three physiological parameters, $x_{1}, x_{2}$ and $x_{3}$ which produce hypoglycemia in a T1DM patient. Based on these domains, $n$ rules in the following form can be extracted.

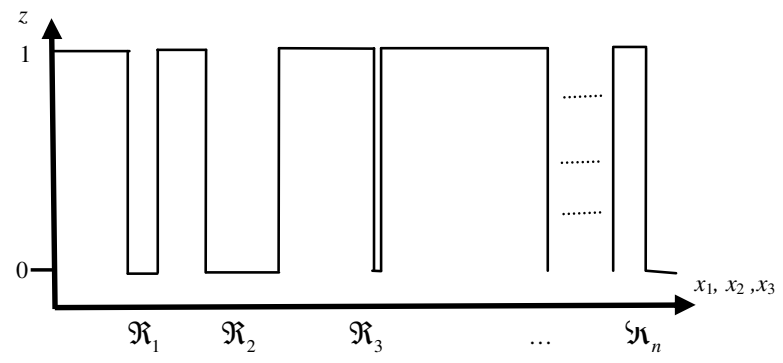

Figure 2 An illustration of the extract rules represent the domain of the physiological parameters

In this section, a knowledge discovery unit is proposed to extract rules from the diagnosis unit. First, a set of data regarding the relationship between the three physiological parameters and hypoglycemia is generated by the diagnosis unit. Then, the rules are developed by the genetic algorithm, in order to form a conjunction hypoglycemia with the true recommended domains of the three physiological parameters. Based on the knowledge discovery system, informative rules involving a domain of three physiological parameters with respect to the hypoglycemia can be extracted from the data sets. The rules generated can be represented as follows:

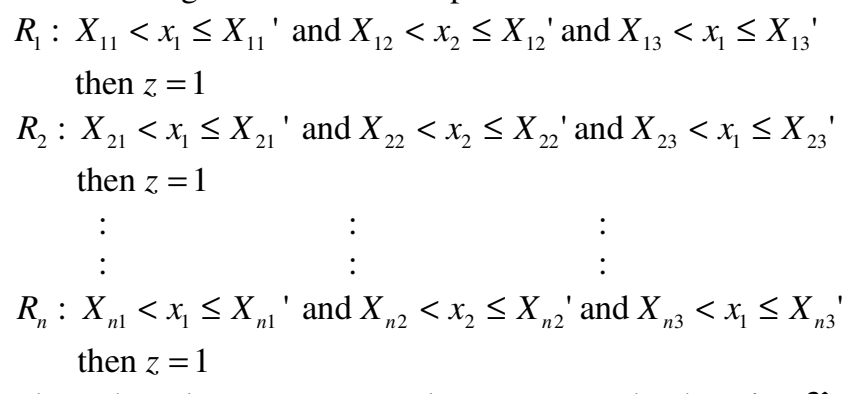
where the rules $R_{1}, R_{2}, \ldots$ and $R_{\mathrm{n}}$ represent the domains $\Re_{1}$, $\mathfrak{R}_{2}, \ldots$ and $\mathfrak{R}_{n}$ of the three physiological parameters in Figure 2 respectively. The pseudocode of the genetic algorithm, which is used to search the parameters (i.e. $X_{11}$, $X_{11}, X_{12}, X_{12}, X_{13}, X_{13}, X_{21}, X_{21}{ }^{\prime}, X_{22}, X_{22}, X_{23}, X_{23}, \ldots$, $X_{n 1}, X_{n 1}, X_{n 2}, X_{n 2}, X_{n 3}, X_{n 3}$ ') on the $n$ rules (i.e. $R_{1}, R_{2}, \ldots$ and $R_{\mathrm{n}}$ ), is shown as follows:

$n=1 ; / *$ where $n$ is the number of rules

\{

$$
n=n+1
$$

Initialize $\Omega(t)=\left[\alpha_{1}(t), \alpha_{2}(t), \ldots \alpha_{P O P}(t)\right]$

Evaluate all $\alpha_{k}(t)$ according to the fitness function defined in equation (3)

while $\left(t<T_{g e n}\right)$ do $/ * T_{g e n}$ is the predefined number of generations

\{

Parent Selection $\Omega(t+1)=\left[\alpha_{1}(t), \alpha_{2}(t), \ldots \alpha_{P O P}(t)\right]$

Crossover $\Omega(t+1)$

Mutation $\Omega(t+1)$

Evaluate all $\alpha_{k}(t+1)$ according to the fitness function defined in equation (3)

Reinsert $\Omega(t)$ by $\Omega(t+1)$

$t=t+1$

\} 
Select the best $\alpha_{k}(t+1)$ among all strings to be the rules extracted from the neural network based classification unit

\} While (the accuracy of the rules is satisfactory)

*/ The algorithm terminates if a string which $\varsigma>0.75$ and $\kappa>0.5$

*/ can achieve and is found.

In the genetic algorithm, $n$ is the number of rules extracted from the neural network. The larger $n$ is, the more likely that the data generated by the neural network will be covered by the rules, and better accuracy is more likely to be achieved. However, a longer computational time is required to search for the optimal parameters of the rules. Therefore, the value of $n$ is initially set at 1 . If no satisfied rule can be found by the genetic algorithm, $n$ is incremented by 1 before the next genetic algorithm run. With an increased $n$, a better accuracy rate is more likely to be achieved.

The genetic algorithm first creates a random initial population $\Omega(t)$ of strings $\left[\alpha_{1}(t), \alpha_{2}(t), \ldots \alpha_{P O P}(t)\right]$ with $t=0$, while $P O P$ is the number of strings of the population. The string $\alpha_{\mathrm{i}}(t)$ is represented as the parameters of the $n$ rules and can be denoted by:

$\alpha_{\mathrm{i}}(t)=$

$\left\{X_{11}{ }^{i}(t), X_{11}{ }^{i}(t)^{\prime}, X_{12}{ }^{i}(t), X_{12}{ }^{i}(t)^{\prime}, \quad X_{13}{ }^{i}(t), X_{13}{ }^{i}(t)^{\prime}, \ldots \ldots\right.$, $\left.X_{n 1}{ }^{i}(t), X_{n 1}{ }^{i}(t)^{\prime}, X_{n 2}{ }^{i}(t), X_{n 2}{ }^{i}(t)^{\prime}, X_{n 3}{ }^{i}(t), X_{n 3}{ }^{i}(t)^{\prime}\right\}$

where all $X_{j k}{ }^{i}(t)$ and $X_{j k}{ }^{i}(t)^{\prime}$ with $j=1,2, \ldots, n ; k=1,2,3$ and $X_{j k}{ }^{i}(t)<X_{j k}{ }^{i}(t)^{\prime}$ are randomly generated within the operation domains of three physiological parameters. Then each string is evaluated by the fitness function based on (3) that indicates the accuracy of the rules discovered. The objective of the genetic algorithm is to maximize the accuracy of the rules discovered by searching the values of the parameters of the rules. After assigning an accuracy score to each string, the string selection process uses each string score to determine the selection of potential strings for the next generation. The roulette-wheel approach, which is one of the most common selection methods, is used to select the strings, $\alpha_{1}(t), \alpha_{2}(t), \ldots \alpha_{P O P}(t)$, for the next evolutionary population $\Omega(t+1)$. Then, evolution of the strings is performed by crossover and mutation.

The fitness function of the rule discovery system is used to evaluate how well a rule fits the data samples generated by the neural network based classification unit. Rules need to be evaluated during the training process in order to establish points of reference for the rule discovery system. The fitness function defined in (3) which consider the data sets as correctly classified, lift to be classified, and the wrongly classified ones are discovered. With the higher numbers of $N_{T P}$ and $N_{T N}$, and the lower number of $N_{F P}$ and $N_{F N}$, a better rule is generated. For a comprehensive discussion about rule-quality measures, the reader can refer to [11]. An illustration of a rule generated by the rule discovery system is shown as follows:

if $1.1 \leq x_{1} \leq 1.4$ and $0.2 \leq x_{2} \leq 0.6$ and $-0.3 \leq x_{3} \leq-0.1$ then $z=1$ where $\quad X_{11}(t)=1.10, \quad X_{11}{ }^{\prime}(t)=1.40, \quad X_{12}(t)=0.20$, $X_{12}{ }^{\prime}(t)=0.60, \quad X_{13}(t)=-0.30$, and $X_{13}{ }^{\prime}(t)=-0.10$, are the values from the string of the knowledge discovery unit. To evaluate the fitness of the rule, 4 training data sets as shown in Table 1 are used. Classifications of the training data sets are shown in the last column of Table 1.

- The 1-st data set is classified as $N_{T P}$ class, since $\mathrm{z}=1$, and also all $x_{1}=1.20, x_{2}=0.5$ and $x_{3}=-0.2$ are within the ranges $1.10 \leq x_{1} \leq 1.40,0.2 \leq x_{2} \leq 0.6$ and $-0.3 \leq x_{3} \leq-0.1$ respectively. Therefore, the data set is covered by the rule and is correctly classified;

- The 2-nd data set is classified as $N_{T N}$ class, since $z=0$, and both $x_{2}=0.1$ and $x_{3}=-0.4$ are not within the ranges $0.2 \leq x_{2} \leq 0.6$ and $-0.3 \leq x_{3} \leq-0.1$ respectively. This means the data set is not covered by the rule but differs from the target class;

- The 3-rd data set is classified as $N_{F N}$ class, since $z=0$, and also all $x_{1}=1.30, x_{2}=0.4$ and $x_{3}=-0.25$ are within the ranges $1.10 \leq x_{1} \leq 1.40,0.2 \leq x_{2} \leq 0.6$ and $-0.3 \leq x_{3} \leq-0.1$ respectively. This means the sample is not covered by the rule but matches the rule;

- The 4-th data set is classified as $N_{F P}$ class, as $z=1$, but all $x_{1}=1.05, x_{2}=0.1$ and $x_{3}=-0.2$ are within the ranges,

$1.10 \leq x_{1} \leq 1.4,0.2 \leq x_{2} \leq 0.6$ and $-0.3 \leq x_{3} \leq-0.1$

respectively. Therefore, the data set is not covered by the rule but is wrongly classified as belonging to the target class.

In this example, the number of data sets in all $N_{F N}, N_{F P}$, $N_{T P}$ and $N_{T N}$ classes is 1 . Thus, based on the fitness function (3), the score of accuracy of rule (6) is calculated as:

$$
\begin{aligned}
\text { fitness }= & \lambda \varsigma+(1-\lambda) \kappa \\
& =0.55 \times 0.5+0.45 \times 0.5=0.5
\end{aligned}
$$

Table 1 Training data for rule (6)

\begin{tabular}{|l|l|l|l|l|l|}
\hline $\begin{array}{l}\text { Data } \\
\text { sets }\end{array}$ & $x_{1}$ & $x_{2}$ & $x_{3}$ & $Z$ & Class \\
\hline $1^{\text {st }}$ & 1.20 & 0.5 & -0.2 & 1 & $N_{t p}$ \\
\hline $2^{\text {nd }}$ & 1.15 & 0.1 & -0.4 & 0 & $N_{t n}$ \\
\hline $3^{\text {rd }}$ & 1.30 & 0.4 & -0.25 & 0 & $N_{f n}$ \\
\hline $4^{\text {th }}$ & 1.05 & 0.1 & -0.2 & 1 & $N_{f p}$ \\
\hline
\end{tabular}

After assigning the score of accuracy to each string, the string selection process uses the score of each string to determine the selection of potential strings for the next generation. The approach of roulette-wheel, which is one of the most common selection methods, is proposed to select the strings, $\alpha_{1}(t), \alpha_{2}(t), \ldots \alpha_{P O P}(t)$, to the population $\Omega(t+1)$.

Then, evolution of the strings is performed by crossover and mutation. Intermediate crossover [14], a common crossover operation for real encoding representation, is used in the genetic algorithm. Then, a mutation operation is carried out by randomly changing one or more parameter values in the selected string. The genetic algorithm stops when the best 
string matches the accuracy specified by the medical doctors. Otherwise, the number of rules represented by the strings is incremented by 1 , and the genetic algorithm is restarted with new population strings in which the number of rules represented is incremented. The process continues until the accuracy of a set of rules can match the accuracy specified by the doctors.

It is necessary to mention that a sufficient amount of data is required to be generated by the diagnosis unit in order for the knowledge discovery unit to create rules for representing the characteristics of the diagnosis unit. If a small amount of data is used for creating the rule by the knowledge discovery unit, a misleading rule, which applies to only some of the characteristics of the diagnosis unit, is likely to be created. Such a rule would under-fit the characteristics of the diagnosis unit, producing a large discrepancy between the characteristics of the diagnosis unit and the rule created by the knowledge discovery unit. A satisfactory amount of data must be generated by the diagnosis unit, in order for the knowledge discovery unit to create a rule that fits the characteristics of the diagnosis unit. This would produce only a small discrepancy between the diagnosis unit and the rule created by knowledge discovery unit. To ensure the quality of the rules generated by the knowledge discovery unit is satisfactory, another set of real data is used for the validation. The rules created are considered to be satisfactory if the validation is satisfactory. If unsatisfactory validation is found with the rule set, the performance of the rule set could be improved by generating a larger amount of data using the diagnosis unit.

\section{RESUlTS AND DISCUSSION}

\section{A. T1DM's data}

The data was collected from sixteen T1DM patients ranging in age between $14.6 \pm 1.5$ years, who were selected to participate in the 10-hour overnight hypoglycemia study at the Princess Margaret Hospital for Children in Western Australia, Australia. Each T1DM patient was monitored overnight for the natural occurrence of nocturnal hypoglycemia. The three required physiological parameters, rates of changes of heart rates, $x_{1}$, corrected QT interval of electrocardiogram signal, $x_{2}$, and rates of changes of corrected QT interval, $x_{3}$, were measured; in addition, the actual blood glucose levels, $y$, were measured by a Yellow Spring Instrument. The actual blood glucose profiles for the 16 T1DM children are shown in Figure 3.

The responses of the sixteen T1DM patients exhibited significant changes during the hypoglycemia phase against the non-hypoglycemia phase. The sampling period was around 5 minutes and 35-40 data were collected from each patient. Three hundred and twenty (320) training data sets regarding $y, x_{1}, x_{2}$ and $x_{3}$ were used for developing the proposed hypoglycemic episode diagnosis system, and 100 testing data sets were used for validation purposes.

\section{B. Results}

The following parameter settings suggested by [17] were implemented on the genetic algorithm to train the diagnosis unit discussed in Section II.A: crossover rate $=0.8$; mutation rate $=1 /\left(5 n_{h}+1\right)$, where $5 n_{h}+1$ is the number of variables of a string and $n_{h}$ is the number of hidden nodes on the neural network; number of generations $=1000$; and population size $=$ 500. The work described above was implemented using Matlab programming software. As the genetic algorithm is a stochastic algorithm, different neural networks could be found with different runs. Therefore, the genetic algorithm was run for 30 times, and the best result among the 30 runs was recorded. The sensitivity and the specificity of the optimized neural network with ten hidden nodes, which was found by the genetic algorithm with the best mean fitness, were 0.7930 and 0.6053 respectively. The results are considered to be satisfactory, as the sensitivity is greater than 0.75 and the specificity is greater than 0.5 . Therefore, the diagnosis unit can achieve satisfactory results; hence, patients can be diagnosed correctly if they experience hypoglycemia.

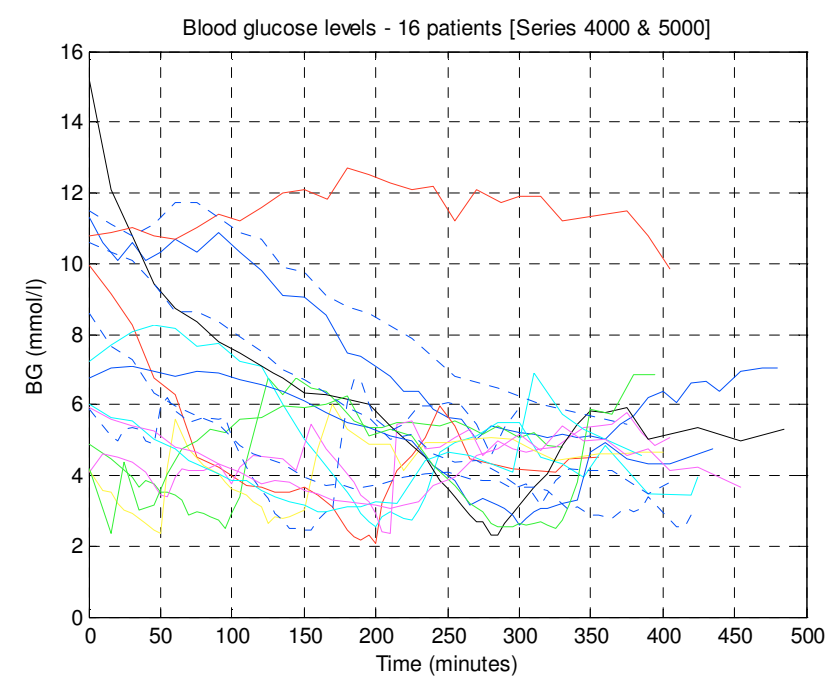

Figure 3 Actual blood glucose level profiles in the 16 T1DM patients

The knowledge discovery unit discussed in Section II.B was implemented using Matlab programming software. The following parameter settings were used: crossover rate $=0.8$; and mutation rate $=1 /\left(6 \times N_{R}\right)$, where the number of variables of the string is $6 \times N_{R}$, and $N_{R}$ is the number of rules extracted by the knowledge discovery system. Based on the 500 data sets regarding to the three physiological parameters and the hypoglycemia generated by the diagnosis unit, the genetic algorithm was run 30 times. The best rule set among the 30 runs was extracted as follows:

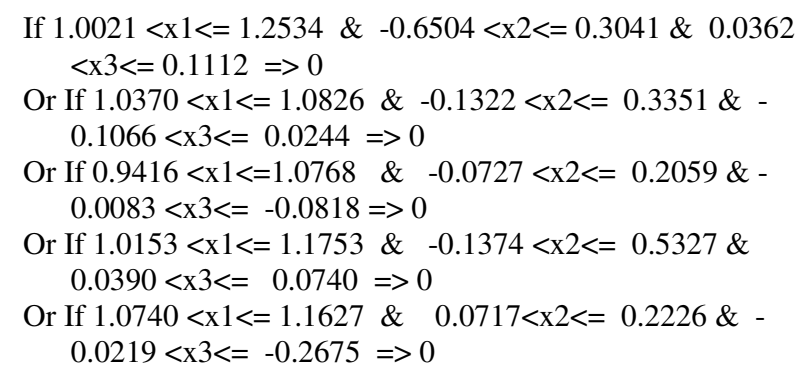


Or If $0.9846<x 1<=1.0341 \&-0.5341<x 2<=-0.7819 \&$ $0.0164<\mathrm{x} 3<=0.0546 \Rightarrow 0$

Or If $1.0679<\mathrm{x} 1<=1.1259 \&-0.5876<\mathrm{x} 2<=0.3072 \&-$ $0.1534<\mathrm{x} 3<=0.0051 \Rightarrow 0$

Or If $1.0807<\mathrm{x} 1<=1.0862 \&-0.0942<\mathrm{x} 2<=-0.1308 \&-$ $0.1377<\mathrm{x} 3<=0.0360 \Rightarrow 0$

Or If $1.0226<x 1<=1.1891 \&-0.2558<x 2<=0.2251 \&-$ $0.1860<\mathrm{x} 3<=0.0138 \Rightarrow 0$

Or If $1.1694<x 1<=1.1731 \& \quad \& \quad-0.1051<x 2<=-0.0349 \&-$ $0.0468<x 3<=-0.0085 \Rightarrow 0$

Or If $1.1229<\mathrm{x} 1<=1.2217 \& \quad 0.0821<\mathrm{x} 2<=0.2235 \&-$ $0.0714<\mathrm{x} 3<=0.1527 \Rightarrow>0$

Or If $1.1555<\mathrm{x} 1<=1.1565 \& 0.072<\mathrm{x} 2<=0.0770 \& \quad$ $0.1939<\mathrm{x} 3<=0.0817 \Rightarrow 0$

Or If $0.9406<\mathrm{x} 1<=1.3289 \&-0.0839<\mathrm{x} 2<=0.2479 \&-$ $0.0752<\mathrm{x} 3<=0.0763=>0$

The sensitivity for the rule set was found to be 0.7047 and the specificity for this was 0.5427 , when the data generated by the diagnosis unit was used as the inputs of the knowledge discovery unit. To validate the developed rule set, the independent 100 testing data, which were the actual measured data of the T1DM patients, were used. The validated sensitivity and the specificity were found to be 0.7911 and 0.5201 respectively which are larger than the specified ones of 0.75 and 0.5 . Therefore, a satisfactory rule set with satisfactory sensitivity and specificity can be created by the knowledge discovery system. Based on the satisfactory rule set, knowledge can be extracted from the diagnosis unit which is structured in a black-box neural network.

\section{CONCLUSION}

In this paper, a hypoglycemic episode diagnosis system is developed to determine the presence of hypoglycemic episodes based on the TIDM patients' physiological parameters, rate of change of heart rate, corrected QT interval of electrocardiogram signal, and rate of change of corrected QT interval. It consists of two units: a) a diagnosis unit is used to perform diagnosis of hypoglycemic episodes in T1DM patients; and b) a knowledge discovery unit is used to generate a set of rules, which describe the domains of physiological parameters for which hypoglycemic episodes occur.

The hypoglycemic episode diagnosis system was developed based on 420 T1DM patients' data sets which were collected from 16 T1DM patients by using the genetic algorithm. Three hundred and twenty (320) data sets were used to develop the hypoglycemic episode diagnosis system and 100 data sets were used to validate its performance. Results show that the sensitivity and specificity were found to be $79.30 \%$ and $60.53 \%$ respectively which are considered reasonable. Explicit rules can be extracted from the knowledge discovery unit based on the proposed genetic algorithm. It is found that the extracted rules can achieve sensitivity of $79.11 \%$ and specificity of $52.01 \%$, both of which are satisfactory. The hypoglycemic episode diagnosis system compensates for the limitations of the traditional neural network approaches which cannot generate implicit information.

\section{REFERENCES}

[1] Aruna P., Puviarasan N., \& Palaniappan B. (2007). Diagnosis of gastrointestinal disorders using DIAGNET. Expert Systems with Applications, 32, 329-335, 2007.

[2] Carvalho D.R., \& Freitas A.A. (2000). A hybrid decision tree/genetic algorithm for coping with the problem of small disjoints in data mining. Proceedings of Conference of Genetic and Evolutionary Computation, 1061-1068, 2000.

[3] Chang C.L., \& Chen C.H. (2009). Applying decision tree and neural network to increase quality of dermatologic diagnosis. Expert Systems with Applications, 36, 4035-4041, 2009.

[4] Chang C.L., \& Hsu M.Y. (2009). The study that applies artificial intelligence and logistic regression for assistance in differential diagnostic of pancreatic cancer. Expert Systems with Applications, 36, 10663-10672, 2009.

[5] Cho B.H., Yu H., Kim K.W., Kim T.H., Kim I.Y. \&. Kim S.I. (2008). Application of irregular and unbalanced data to predict diabetic nephropathy using visualization and feature selection methods. Artificial Intelligence in Medicine, 42(1), 37-53.

[6] Chu A., Ahn H., Halwan B., Kalmin B., Artifon E.L.V., Barkun A., Lagoudakis M.G. \& Kumar A. (2008). A decision support system to facilitate management of patients with acute gastrointestinal bleeding. Artificial Intelligence in Medicine, 42(3), 247-259.

[7] Das R., Turkoglu I. \& Sengur A. (2009). Effective diagnosis of heart disease through neural networks ensembles. Expert Systems with Applications, 36, 7675-7680.

[8] DCCT Research Group. (1993). The effect of intensive treatment of diabetes on the development and progression of long-term complications in IDDM. The New England Journal of Medicine, 329, 977-986.

[9] DCCT Research Group. (1995). Adverse events and their association with treatment regimens in the Diabetes Control and Complications Trial. Diabetes Care, 18, 1415-1427.

[10] Gil D., Johnson M., Chamizo J.M.G.., Paya A.S. \& Fernandez D.R. (2009). Application of artificial neural networks in diagnosis of urological dysfunctions. Expert Systems with Applications, 36, 57545760.

[11] Hand D.J., (2001). Principles of Data Mining, MIT Press.

[12] Harris N.D., Baykouchev S.B., Marques L.B., Cochrane T., George E., Heller S.R., \& Ward J.D., (1996). A portable system for monitoring physiological responses to hypoglycaemia. Journal of Medical Engineering and Technology, 20(6), 196-202.

[13] Mantzaris D., Anastassopoulos G..A. \& Gardikis S., (2008). A nonsymbolic implementation of abdominal pain estimation in childhood. Information Sciences, 178, 3860-3866.

[14] Muhlenbein H. \& Voosen D.S. (1993). Predictive models for the breeder genetic algorithm: I. Continuous parameter optimization. Evolutionary Computation, 1(1), 25-49.

[15] Pickup J.C. (2000). Sensitivity glucose sensing in diabetes. Lancet, 355, 426-427.

[16] Reggia J.A., \& Sutton S.S. (1988). Self-processing networks and their biomedical implications. Proceedings of the IEEE, 76(6), 580-592.

[17] Schaffer J., Caruana R., Eshelman L., \& Das R. (1989). A study of control parameters affecting online performance of genetic algorithms for function optimization. Proceedings of the 3rd International Conference on Genetic Algorithms, 51-60.

[18] Seber G..A.F. (2003). Linear regression analysis, Wiley.

[19] Yale J.F. (2004). Nocturnal hypoglycemia in patients with insulintreated diabetes. Diabetes Research and Clinical Practice, 65, 41-46. 